\title{
Hamartomatous Inverted Polyp Successfully Treated by Endoscopic Submucosal Dissection
}

\author{
Masaru Odashima ${ }^{1}$, Michiro Otaka ${ }^{1}$, Hiroshi Nanjo ${ }^{2}$, Mario Jin ${ }^{1}$, Youhei Horikawa ${ }^{1}$, \\ Tamotsu Matsuhashi ${ }^{1}$, Reina Ohba ${ }^{1}$, Shigeto Koizumi ${ }^{1}$, Nobukatsu Kinoshita ${ }^{1}$, \\ Taiji Takahashi ${ }^{1}$, Hitoshi Shima ${ }^{3}$ and Sumio Watanabe ${ }^{1}$
}

\begin{abstract}
We report a case of gastric hamartomatous inverted polyps that are a rare histological type of gastric polyp and difficult to diagnose. Gastric submucosal tumor was detected by upper gastrointestinal X-ray series in 37-year-old man. Endoscopy revealed a submucosal tumor (SMT), which eroded with a depression on its surface in the fornix. Endoscopic ultrasonography showed a heterogeneous tumor in the third layer. Endoscopic submucosal dissection (ESD) was performed to resect the tumor completely. The pathological diagnosis was a gastric hamartomatous inverted polyp. The patient was later discharged without any complications. Hamartomatous inverted polyps without a stalk are classified as the SMT type because the tumor is inverted down growth into the submucosal layer, otherwise polyps with a stalk are classified as the polyp type. All of the polyps were resected endoscopically, however, surgical resection was performed for those of the SMT type, because it is difficult to remove this type completely by en-block resection using conventional EMR technique. ESD method may be indicated for SMT-type hamartomatous inverted polyps.
\end{abstract}

Key words: hamartomatous inverted polyp, endoscopic submucosal dissection

(DOI: 10.2169/internalmedicine.47.0360)

\section{Introduction}

A usual type of gastric polyp, mimicking a submucosal tumor, has been described as a hamartomatous polyp $(1,2)$. The polyps consist of the overlying gastric mucosa and submucosal component that is characterized by proliferation of pseudo-pylorus glands, cystic glands, and smooth muscle bundles $(1,2)$. Although these polyps are considered to be hamartomatous or ectopic in nature, their histogenesis remains to be solved. To make an accurate diagnosis of gastric hamartomatous polyps is difficult preoperatively because of their rarity. Here, we report a case of gastric hamartomatous inverted polyp of submucosal tumor (SMT) type, which was successfully resected by ESD technique without any complication.

\section{Case Report}

A 37-year-old man was admitted to Akita University Hospital for investigation and treatment of a submucosal tumor (SMT), over $2 \mathrm{~cm}$ in diameter, which had been detected by an upper gastrointestinal endoscopy. He was asymptomatic and his family history was unremarkable for gastrointestinal disorders. No pigmentation was found in the mouth and lips. Physical examination and laboratory data on admission showed no signs of abnormality. Both upper gastrointestinal X-ray series and endoscopy showed an SMT in the fornix, with erosion and a depression on its surface (Fig. 1a). Endoscopic ultrasonography revealed a heterogeneous tumor, and most of the tumor was located in the third (submucosal) layer (Figs. 1b, 1c). These findings suggested possibility of malignant neoplasm. But a biopsy specimen from the bottom of the depression showed gastric mucosa with hyper-

${ }^{1}$ Department of Gastroenterology, Akita University School of Medicine, Akita, ${ }^{2}$ Department of Clinical Pathology, Akita University School of Medicine, Akita and ${ }^{3}$ Ogawa Clinic, Akita

Received for publication June 2, 2007; Accepted for publication October 29, 2007

Correspondence to Dr. Masaru Odashima, odashima@doc.med.akita-u.ac.jp 

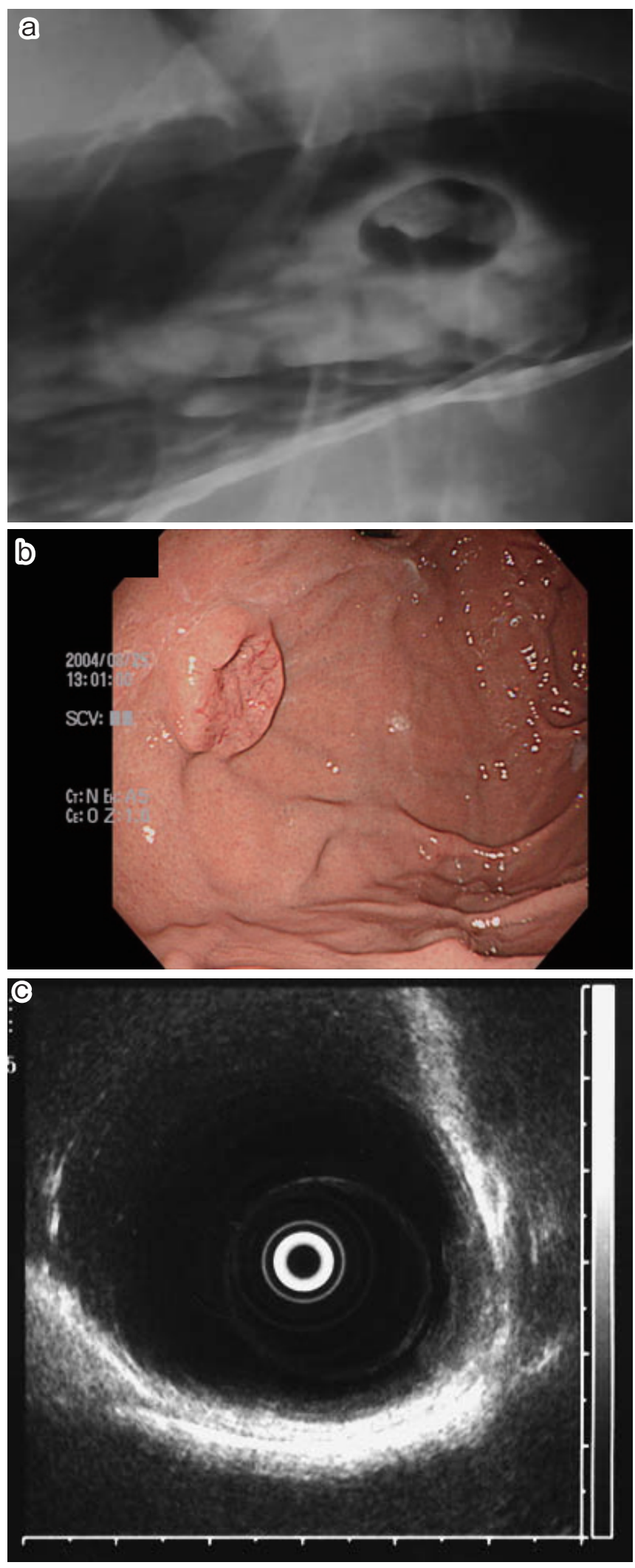

Figure 1. a: Upper gastrointestinal X-ray series showed a submucosal tumor in the fornix.

b: Upper gastrointestinal endoscopy showed erosion and depression at the top of the submucosal tumor.

c: Endoscopic ultrasonography (7.5 MHz) showed a heterogeneous tumor, most of which was located in the third (submucosal) layer.

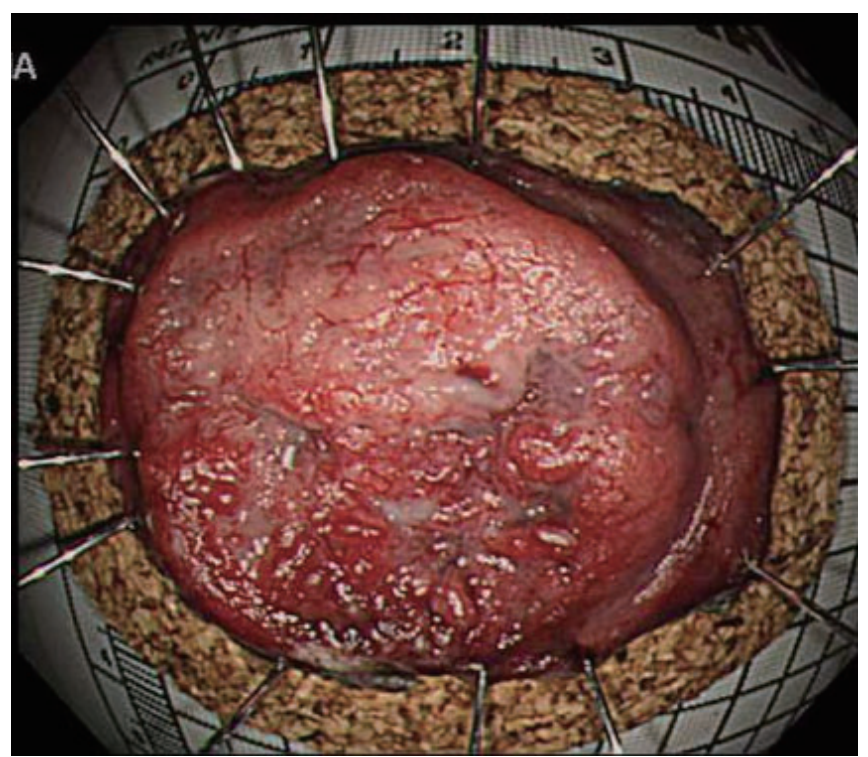

Figure 2. Operative specimen. The tumor, which was 2.5 cm in diameter, was resected completely.

plastic change. Because it was difficult to make a definitive diagnosis based on these findings, we considered that resection was necessary to make an accurate diagnosis and select optimal treatment. However, we thought that it was impossible to resect the tumor with conventional endoscopic mucosal resection (EMR) technique, such as strip biopsy and endoscopic mucosal resection with a cap fitted endoscope. Therefore, we performed EMR using endoscopic submucosal dissection (ESD) technique to remove the tumor completely by one segment resection. ESD achieved complete resection of the tumor with $2.5 \mathrm{~cm}$ in diameter (Fig. 2). Histologically, the tumor was characterized by submucosal proliferation of the gland and cystic structures without cytological atypia. The surface of the polyp was covered with gastric mucosa of fundic or pyloric gland type. The glandular structures consisted of various types of lining cells, including pyloric or mucous-neck cell type, surface mucous (foveolar) cell type, and parietal-like cells (Fig. 3). The submucosal glandular or cystic elements were connected with the overlying gastric mucosa through a defect of the muscularis mucosa, suggesting that this polyp may have been formed by the heterotopic inverted down growth of mucous glands into the submucosa. The patient was discharged 5 days after EMR without any complication.

\begin{tabular}{l} 
Discussion \\
\hline Gastric hamartomatous inverted polyps are characterized \\
by marked submucosal glandular proliferation associated \\
with cystic dilatation. The main lesion is located in submu- \\
cosa or the inside of muscularis mucosae. They are different \\
to hamartomas, which are non-neoplastic formation in which \\
tissue indigenous to that organ are abnormally intermixed, \\
usually with preponderance of one or more than one type of \\
tissue (1). The predominance elements if hamartomatous are
\end{tabular}



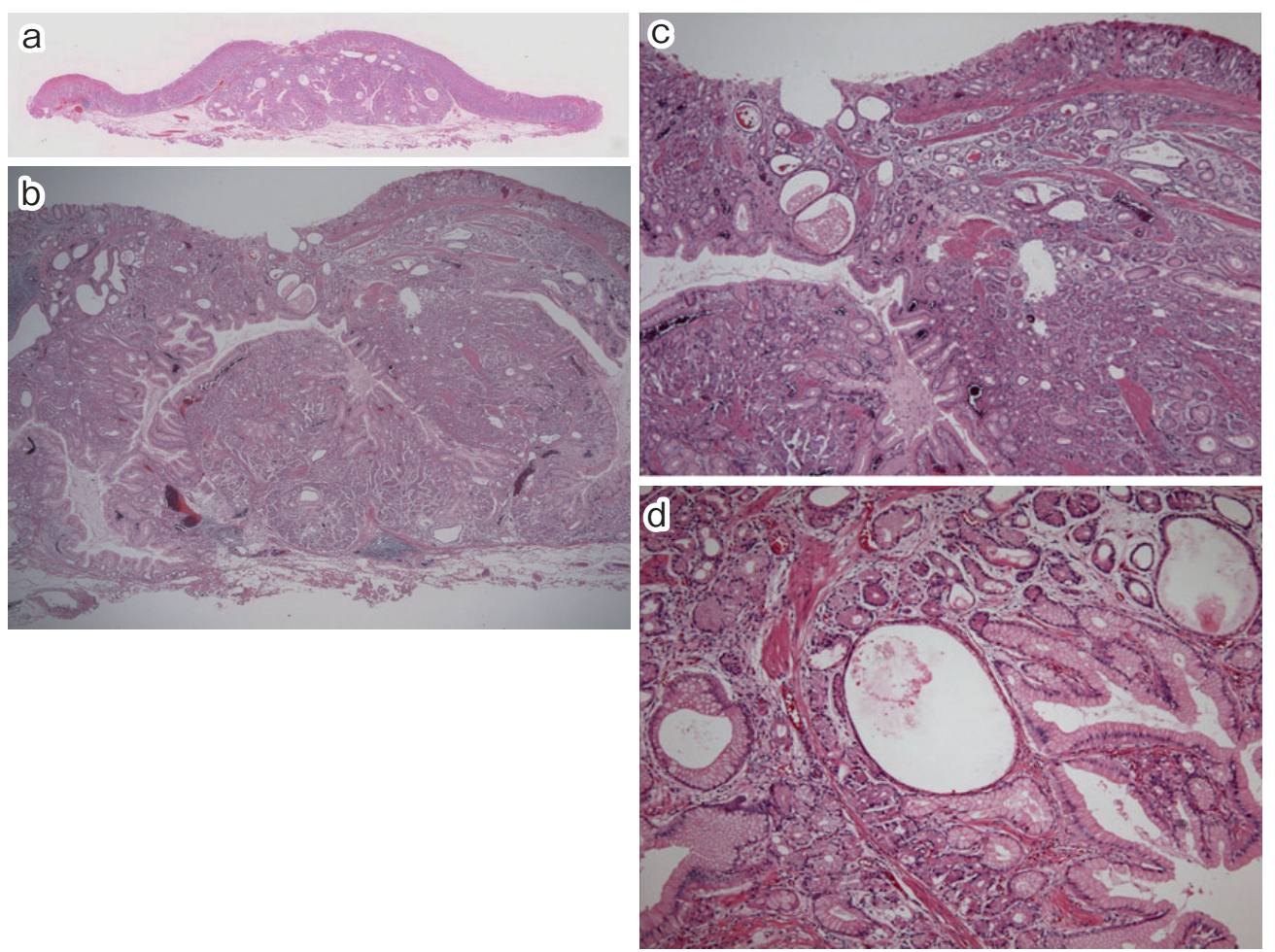

Figure 3. a: Microscopy of the endoscopic resection specimen revealed hypertrophy of the tumor component in the submucosal layer (Hematoxylin and Eosin staining). b, c, d: Glands with cyst formation are located in the submucosal layer. The muscularis mucosa communicated with and extended downwards into the submucosal layer.

connective tissue derivatives, including nerve elements, vasoformative tissue, smooth muscle, and lamina propria (1, 2). Gastric hamartomatous polyps are rare, being found in less than $1 \%$ of all gastric polyps $(3,4)$. Previous reports have shown that there is not any gender preponderance and these lesions were considered to be acquired rather than congenital, because they occurred in older adults (5). Gastric hamartomatous polyps are usually asymptomatic and found incidentally in patients. However, they may occasionally manifest as an anemia secondary to chronic blood loss or as an intestinal obstruction. It is noteworthy that gastric hamartomatous polyps are benign disease pathologically but about $20 \%$ of gastric hamartomatous polyps coexisted with gastric adenocarcinoma (6). Therefore, the diagnosis and treatment of these polyps remain important problems. Previous papers described that it is difficult to make diagnosis before treatment (6). The treatment of gastric hamartomatous polyps was determined by tumor features and size. There are two types of hamartomatous inverted polyps;those without a stalk, which are the "SMT type" because the tumor is inverted into the submucosal layer, and those with a stalk, which are the "polyp type," While all polyps with a stalk were endoscopically resected, all polyps without a stalk (SMT type) were surgically resected because endoscopic resection of polyps without a stalk resulted incomplete resection in previous reports (5). According to these data, previous reports recommended surgical resection for gastric hamartomatous polyps of the SMT type to remove the tumor completely. In the present case, the submucosal tumor of the stomach was lager than $2.0 \mathrm{~cm}$ in diameter. However, EMR by the conventional method requires sophisticated techniques, it has only been reliable for obtaining specimens of lesions measuring $10 \mathrm{~mm}$ or less in one piece resection (7, 8). Therefore, it was considered to be difficult to resect the tumor completely by the conventional EMR method. However, since the development of a new technique using an insulation-tipped diathermic knife (IT knife), it has been found that circumferential mucosal incisions performed with IT knife around lesions before snaring, increases en bloc resection rates, suggesting the possibility of expanding the indications for EMR (9). And, many reports revealed that if the lesion is $10 \mathrm{~mm}$ or greater, ESD method has been selected to prevent piece-meal resection, which is liable to provide insufficient tissue for accurate pathologic diagnosis $(10,11)$. Furthermore, endoscopic resection is comparable in many respects to conventional surgery, with the advantages of being less invasive and more economical (12). Thus, we successfully treated this tumor using ESD method. We recommend that SMT-type hamartomatous inverted polyps lager than $2.0 \mathrm{~cm}$ in diameter be treated by EMR using the ESD method. 


\section{References}

1. Ming S. Tumors of the esophagus and stomach. In: Tumors of the Stomach. 2nd, Ed. Armed Forces Institute of Pathology, 1973: 124-143.

2. Katz LB, Tenembaum MM, Kree I. Gastric hamarmatous polyp in the absence of familial polyposis. Mount Sinai J Med 49: 426429, 1982.

3. Iida M, Yao T, Watanabe $H$, Itoh H, Iwashita A. Fundic gland polyposis in patients without familial adenomatous coli: its incidence and clinical features. Gastroenterology 86: 1437-1442, 1984.

4. Sipponen P, Siurala M. Cystic "hamartomatous" epithelial polyps of the stomach. Acta Hepatogastroenterol 25: 380-383, 1978.

5. Aoki M, Yoshida M, Saikawa Y, et al. Diagnosis and treatment of a gastric hamartomatous inverted polyp: report a case. Surg Today 34: 532-536, 2004.

6. Yamashita M, Hirokawa M, Nakasono M, et al. Gastric inverted hyperplastic polyp. Report of four cases and relation to gastritis cystica profunda. APMIS 110: 717-723, 2002.

7. Inoue H, Takeshita K, Hori H, Muraoka Y, Yoneshima H, Endo
M. Endoscopic mucosal resection with a cap fitted panendoscope for esophagus, stomach, and colon mucosal lesions. Gastrointest Endosc 39: 58-62, 1993.

8. Matsushita M, Hajiro K, Okazaki K, Takakuwa H. Endoscopic mucosal resection of gastric tumors located in the lesser curvature of the upper third of the stomach. Gastrointest Endosc 45: 512$515,1997$.

9. Gotoda T, Kondo H, Ono H, et al. A new endoscopic mucosal resection procedure using an insulation-tipped electrosurgical knife for rectal flat lesions: report of two cases. Gastrointest Endosc 50: 560-563, 1999.

10. Miyazaki S, Gunji Y, Aoki T, et al. High en bloc resection rate achieved by endosopic mucosal resection with IT knife for early gastric cancer. Hepatogastroenterology 52: 954-958, 2005.

11. Ono H, Kondo H, Gotoda $\mathrm{T}$, et al. Endoscopic mucosal resection for treatment of early gastric cancer. Gut 48: 225-229, 2001.

12. Gotoda T. Endoscopic resection of early gastric cancer. Gastric Cancer 10: 1-11, 2007.

(C) 2008 The Japanese Society of Internal Medicine http://www.naika.or.jp/imindex.html 